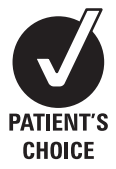

- Additional tables are published online only. To view these files please visit the journal online (http://jnnp.bmj. com/content/83/2.toc).

${ }^{1}$ Division of Stroke and Critical Care, Department of Neurology, University of Southern

California, Los Angeles, California, USA

2Department of Neurology, Rancho Los Amigos National Rehabilitation Center, Downey, California, USA

${ }^{3}$ Department of

Biomathematics, University of California at Los Angeles, Los Angeles, California, USA

${ }^{4}$ Stroke Center and Department of Neurosciences, University of California at San Diego, San

Diego, California, USA

Correspondence to

Dr A Towfighi, 1510 San Pablo Street, HCC 643, Los Angeles, CA 90033, USA;

towfighi@usc.edu

Received 3 September 2011 Accepted 11 September 2011 Published Online First

21 October 2011

\title{
Impact of a healthy lifestyle on all-cause and cardiovascular mortality after stroke in the USA
}

\author{
Amytis Towfighi, ${ }^{1,2}$ Daniela Markovic, ${ }^{3}$ Bruce Ovbiagele $^{4}$
}

\section{ABSTRACT}

Background Little is known about the effects of a healthy lifestyle on mortality after stroke. This study assessed whether five healthy lifestyle factors had independent and dose dependent associations with allcause and cardiovascular mortality after stroke.

Methods In a nationally representative sample of the US population ( $n=15299)$ with previous stroke $(n=649)$ followed from survey participation (1988-1994) through to mortality assessment (2000), the relationship between five factors (eating $\geq 5$ servings of fruits/ vegetables per day, exercising $>12$ times/month, having a body mass index of $18.5-29.9 \mathrm{mg} / \mathrm{kg}^{2}$, moderate alcohol use [1 drink/day for women and 2 drinks/day for men] and not smoking) and all-cause and cardiovascular mortality was assessed.

Results Mean age was 67.0 years (SE 1.1 years) and $53 \%$ were women. After adjusting for covariates, abstaining from smoking (HR 0.57, $\mathrm{Cl} 0.34$ to 0.98 ) and exercising regularly (HR $0.66, \mathrm{Cl} 0.44$ to 0.99 ) were associated with lower all-cause mortality but no individual factors had independent associations with cardiovascular mortality. All-cause mortality decreased with higher numbers of healthy behaviours $(1-3$ factors vs none: $\mathrm{HR} 0.12, \mathrm{Cl} 0.03$ to $0.47 ; 4-5$ factors vs none: HR 0.04 , Cl 0.01 to $0.20 ; 4-5$ factors vs $1-3$ factors: HR 0.38, $\mathrm{Cl} 0.22$ to 0.66; trend $p=0.04$ ). Similar effects were observed for cardiovascular mortality $(4-5$ factors vs none: HR $0.08, \mathrm{Cl} 0.01$ to $0.66 ; 1-3$ factors vs none: HR $0.15, \mathrm{Cl} 0.02$ to $1.15 ; 4-5$ factors vs $1-3$ factors: HR 0.53, Cl 0.28 to 0.98 ; trend $p=0.18$ ).

Conclusions Regular exercise and abstinence from smoking were independently associated with lower all-cause mortality after stroke. Combinations of healthy lifestyle factors were associated with lower all-cause and cardiovascular mortality in a dose dependent fashion.

\section{BACKGROUND}

Stroke survivors have a higher mortality risk than the general population, even years after the index event. ${ }^{1-3}$ Studies have revealed that adherence to a combination of healthy lifestyle practices is associated with reduced stroke incidence ${ }^{4}$ and mortality risk in the general population ${ }^{6-11}$; however, little is known about the effect of a healthy lifestyle on risk of death after stroke.

The objectives of this study were twofold: (1) to assess whether each of the individual five healthy lifestyle factors were independently associated with lower all-cause and cardiovascular mortality after stroke and (2) to investigate whether higher numbers of healthy lifestyle behaviours were associated with a greater survival benefit.

\section{METHODS}

\section{Population for study}

The National Health and Nutrition Examination Survey (NHANES) are cross sectional samples of the US civilian, non-institutionalised population conducted by the National Center for Health Statistics, a branch of the Centers for Disease Control and Prevention. The protocols for conduct were approved by the National Center for Health Statistics institutional review board and informed consent was obtained from all participants. ${ }^{12}$ The sampling plan followed a complex, stratified, multistage, probability cluster design, with oversampling of non-Hispanic blacks, Mexican Americans and the elderly, to enhance the precision of prevalence estimates in those groups. Details of the survey design and examination procedures have been previously published. ${ }^{12}$

In the third NHANES (NHANES III), conducted from 1988 to 1994, 33199 adults were interviewed. The study outcomes-all-cause and cardiovascular mortality-were recorded from NHANES III mortality follow-up data, which relied on a probabilistic match between NHANES III and National Death Index death certificate records. Mortality records were available for 20024 of 20050 adults who completed both interviews and medical examinations. Mortality assessments, including cause specific mortality and mortality dates, were conducted from baseline interview to 31 December 2000. Cause specific mortality was coded using the ninth revision of the International Classification of Diseases, Injuries and Causes of Death (ICD-9) for deaths occurring between 1988 and 1998 and the 10th revision (ICD-10) for deaths occurring between 1999 and 2000. The Underlying Cause of Death 113 Groups All Years (UCOD-113) variable recoded all deaths prior to 1999 coded under ICD-9 guidelines into comparable ICD-10 codes. ${ }^{13}$

Of 649 persons with a self-reported history of stroke, 164 were assigned negative survey weights and were excluded by NHANES. We followed the NHANES survey design by excluding these individuals, leaving 485 persons for the analysis. All 485 persons had mortality follow-up data. Of these 485 persons, $97(20 \%)$ had missing values for the covariates, leaving 388 persons for the complete case analysis. We compared the healthy lifestyle factors and covariates among the complete set $(n=388)$ versus the incomplete set $(n=97)$ to assess qualitative differences between groups. 


\section{Primary outcome variable}

The primary outcome variable was all-cause mortality, analysed as a time to event outcome recorded in months (event was deceased from all causes versus alive).

\section{Secondary outcome variable}

The secondary outcome variable was cardiovascular mortality, analysed as a time to event outcome recorded in months (event was deceased due to cardiovascular causes versus alive while adjusting for competing non-cardiovascular causes). Cardiovascular deaths included deaths from any heart disease, cerebrovascular cause, atherosclerosis or hypertension (UCOD-113 codes 054-074). Stroke mortality (deaths from any cerebrovascular cause, UCOD-113 code 070) was not used as an outcome as it was too rare to formally control for covariates.

\section{Primary predictor variables}

Definitions of healthy lifestyle behaviours were consistent with a previous study of healthy lifestyle practices/factors: eating $\geq 5$ servings of fruits/vegetables/day, exercising $>12$ times/month, body mass index (BMI) of 18.5-29.9 mg/ $\mathrm{kg}^{2}$, drinking alcohol in moderation (1 drink/day for women and 2 drinks/day for men) and not smoking. ${ }^{14}$ These variables have been evaluated in prior studies $^{6}{ }^{14}$ and are endorsed by national guidelines on stroke prevention. ${ }^{15} 16$ BMI was calculated from height and weight $\left(\mathrm{kg} / \mathrm{m}^{2}\right)$ measured using standardised protocols. The other variables were obtained by self-report.

Diet

Although studies have used different definitions of a healthy diet, several studies used fruit/vegetable intake, ${ }^{17-19}$ and the American Heart Association recommends five servings of fruits/vegetables/day as part of a healthy diet. ${ }^{20}$

\section{Exercise}

Physical activity frequency was determined according to participation in leisure time physical activities within the previous month, including walking, jogging or running, riding a bicycle, swimming, aerobic exercise or other similar activities. Current guidelines recommend $\geq 30$ min of moderate intensity activity $\geq 5$ days/week ${ }^{21}$; however, a cardiovascular benefit is evident with as little as $1 \mathrm{~h}$ of running or $30 \mathrm{~min}$ of weight training per week. ${ }^{22}$ Physical activity was divided into two frequency groups (0-12 and $>12$ times/month), consistent with national recommendations at the time of NHANES 1988-1994. ${ }^{23}$

Body mass index

Although a BMI of $18.5-24.9 \mathrm{~kg} / \mathrm{m}^{2}$ is considered optimal, there is no excess mortality risk for overweight individuals (BMI $25-29.9 \mathrm{~kg} / \mathrm{m}^{2}$ ) compared with normal weight individuals (BMI $\left.18.5-24.9 \mathrm{~kg} / \mathrm{m}^{2}\right)^{24}$; therefore, a more liberal range of $18.5-29.9 \mathrm{~kg} / \mathrm{m}^{2}$ was used for this study.

\section{Alcohol use}

Moderate alcohol consumption was defined as 1 drink/day for women and 2 drinks/day for men, according to current USDA guidelines. $^{25}$

\section{Covariates}

Covariates assessed were: age, sex, race/ethnicity, history of myocardial infarction (MI), hypertension, diabetes mellitus (DM), hypercholesterolaemia, hypertriglyceridaemia and low level of high density lipoprotein (HDL) cholesterol.
Race/ethnicity was obtained by self-report. History of MI was defined by self-reported physician diagnosis. Hypertension was defined by self-reported physician diagnosis, self-reported current medical therapy or mean of the first three blood pressure readings $>140 \mathrm{~mm} \mathrm{Hg}$ systolic or $90 \mathrm{~mm} \mathrm{Hg}$ diastolic. DM was defined by self-reported physician diagnosis, self-reported current medical therapy (insulin or oral agents) or glycosylated haemoglobin level $>7 \%$. Hypercholesterolaemia was defined by self-reported physician diagnosis, self-reported current medical therapy or total cholesterol level $>200 \mathrm{mg} / \mathrm{dl}$. Hypertriglyceridaemia was defined as triglyceride level $>150 \mathrm{mg} / \mathrm{dl}$. Low HDL level was defined as HDL $<50 \mathrm{mg} / \mathrm{dl}$ in women and $<40 \mathrm{mg} / \mathrm{dl}$ in men.

\section{Statistical analysis}

Weighted estimates were applied to the descriptive prevalence analysis using NHANES mobile examination centre examined sample weight values. These weights adjusted for the differential probabilities of selection and non-response in the survey sample. To account for NHANES clustering, stratification and unequal weights on the Cox regression models below, the primary sampling unit variable, the stratification variable and the weight variable were adjusted for in the analysis. Statistical hypotheses were tested using $p<0.05$ as the level of statistical significance.

\section{Bivariate analysis}

To assess the bivariate relationship between each covariate and all-cause mortality, the Cox regression model was used, adjusting for the survey design variables. For cardiovascular mortality, the Cox model was expanded to a competing risks Cox model as non-cardiovascular mortality is a simultaneous competing risk.

\section{Multivariable analyses}

The multivariable Cox regression and competing risks models were used to assess the simultaneous influence of all five healthy lifestyle factors on risk of all-cause and cardiovascular mortality, respectively, while adjusting for covariates. The final multivariable models excluded variables that were not significant at the $p<0.25$ level using backwards selection. The relation between number and combination of health factors (versus none) and mortality outcomes was assessed using linear contrasts under the above additive models. As some excluded variables had missing data, the sample sizes for the final multivariable models increased slightly, with 428 individuals in the all-cause mortality model and 419 subjects in the cardiovascular mortality model.

To assess whether a higher number of health factors was associated with improved mortality outcomes, we divided the sample into groups based on number of health factors followed $(0,1-3,4-5)$ and carried out a Cox regression analysis adjusting for demographic and clinical factors. Those who followed five health factors were rare and were thus grouped with those who followed four health factors. Since the analysis indicated that those who followed one, two or three health factors had similar mortality outcomes in the above Cox model, they were combined into a single category. Adjusted survival curves over time in the above groups were estimated under the above Cox regression model. For cardiovascular mortality, the corresponding cumulative incidence curves over time were constructed under the competing risks regression model after adjusting for the covariates.

Because of the potentially intersecting causal relationships among confounders and primary predictors, several nested 
models were assessed. As exercise and diet likely influence BMI, we considered a model with only BMI as a health factor (without the other four healthy factors) after adjusting for demographic factors (age, sex, race) and clinical factors (the clinical factors in our final multivariable analysis) and a similar model without the clinical factors (hypothesised mediators). In addition, we considered a model with four healthy factors without BMI (hypothesised mediator) after adjusting for demographic and clinical factors and a similar model without the clinical factors (hypothesised mediators). Finally, we considered a model with all variables included. To strengthen the validity of our findings, analyses were performed both on the complete case sample $(n=388)$ and after using single imputation for the missing values $(n=485)$.

\section{RESULTS}

Among all adults with a history of stroke who participated in NHANES 1988-1994, mean age was 67.0 years (SE 1.1 years) and $50 \%$ were women. Table 1 depicts the demographic characteristics, medical comorbidities and lifestyle practices of individuals with a history of stroke. The majority of stroke survivors were white $(79 \%)$, had hypertension $(72.5 \%)$, hypercholesterolaemia (67\%), hypertriglyceridaemia (59\%) and low HDL cholesterol $(52 \%)$. With respect to lifestyle factors, most stroke survivors were non-smokers (75\%), ate 1-4 servings of fruits/vegetables per day $(58 \%)$, had a BMI in the $18.5-29.9 \mathrm{~kg} / \mathrm{m}^{2}$ range $(71 \%)$ and

Table 1 Descriptive summary of population-sample $n=388$ (weighted sample $n=3002561$ )

\begin{tabular}{|c|c|c|c|}
\hline Variable & $\begin{array}{l}\text { Weighted } \\
\text { frequency }\end{array}$ & $\begin{array}{l}\text { Weighted } \\
\text { per cent }\end{array}$ & SE (\%) \\
\hline \multicolumn{4}{|l|}{ Demographics } \\
\hline Female & 1497764 & 49.9 & 3.9 \\
\hline Hispanic & 295480 & 9.8 & 2.6 \\
\hline Black & 328902 & 11.0 & 1.6 \\
\hline White & 2378179 & 79.2 & 2.9 \\
\hline \multicolumn{4}{|l|}{ Medical comorbidities } \\
\hline Hypertension & 2175824 & 72.5 & 3.5 \\
\hline Diabetes mellitus & 761041 & 25.3 & 2.6 \\
\hline Hypercholesterolaemia & 2003838 & 66.7 & 3.4 \\
\hline Hypertriglyceridaemia & 1765326 & 58.8 & 3.1 \\
\hline Low level of high density lipoprotein & 1574440 & 52.4 & 4.7 \\
\hline History of myocardial infarction & 689934 & 23.0 & 3.3 \\
\hline \multicolumn{4}{|l|}{ Lifestyle factors } \\
\hline Non-smoker & 2251751 & 75.0 & 3.4 \\
\hline$\geq 5$ servings fruits/vegetables/day & 1188398 & 39.6 & 4.0 \\
\hline $1-4$ servings fruits/vegetables/day & 1755977 & 58.5 & 3.9 \\
\hline 0 servings fruits/vegetables/day & 58186 & 1.9 & 0.6 \\
\hline Regular exercise & 957260 & 31.9 & 3.5 \\
\hline Body mass index $\geq 30 \mathrm{~kg} / \mathrm{m}^{2}$ & 847054 & 28.2 & 3.9 \\
\hline Body mass index $<18.5 \mathrm{~kg} / \mathrm{m}^{2}$ & 34556 & 1.2 & 0.6 \\
\hline Body mass index $18.5-29.9 \mathrm{~kg} / \mathrm{m}^{2}$ & 2120951 & 70.6 & 3.9 \\
\hline Heavy alcohol intake & 42038 & 1.4 & 0.6 \\
\hline No alcohol intake & 2270615 & 75.6 & 3.6 \\
\hline Moderate alcohol intake & 689908 & 23.0 & 3.6 \\
\hline \multicolumn{4}{|l|}{ No of lifestyle factors followed } \\
\hline 0 & 102432 & 3.4 & 1.3 \\
\hline 1 & 482079 & 16.1 & 2.9 \\
\hline 2 & 1075592 & 35.8 & 3.3 \\
\hline 3 & 879540 & 29.3 & 3.9 \\
\hline 4 & 378197 & 12.6 & 2.7 \\
\hline 5 & 84720 & 2.8 & 1.2 \\
\hline
\end{tabular}

did not drink (76\%). The only differences between the complete and incomplete sets were that individuals in the complete set were more likely to be female and to exercise regularly.

Of the 388 individuals with a history of stroke, 208 persons died, of whom 126 died of cardiovascular causes. After bivariate analysis, healthy factors associated with lower all-cause mortality after stroke included moderate alcohol use (versus none) (HR 0.41, CI 0.22 to 0.76 ) and regular exercise (HR 0.59, CI 0.40 to 0.86 ) (table 2). Abstinence from smoking was associated with higher all-cause mortality; however, this effect only approached significance (HR 1.57, CI 0.98 to 2.52). After bivariate analysis, healthy practices associated with lower cardiovascular mortality after stroke included eating 1-4 servings of fruits/ vegetables/day (versus none) (HR 0.30, CI 0.12 to 0.74), eating $\geq 5$ servings of fruits/vegetables/day (versus none) (HR 0.44 , CI 0.19 to 1.02 ) and moderate alcohol use (versus none) (HR 0.51, CI 0.25 to 1.05 ); however, the latter two variables only approached significance (table 2). Among covariates, increasing age, history of MI, hypertension and DM were associated with higher all-cause and cardiovascular mortality after stroke (table 2).

Regular exercise (HR 0.66, CI 0.44 to 0.99 ) and not smoking (HR 0.57, CI 0.34 to 0.98 ) were independently associated with lower all-cause mortality after adjusting for covariates (table 3 ). The nested multivariable models using the complete dataset and imputed missing variables showed similar results (see supplementary tables 1 and 2 available online only). None of the healthy lifestyle factors independently lowered the risk of cardiovascular mortality after stroke after adjusting for covariates; however, eating 1-4 servings of fruits/vegetables/day (versus none) (HR 0.30, CI 0.08 to 1.08 ) and eating $\geq 5$ servings of fruits/vegetables/day (versus none) (HR 0.30, CI 0.09 to 1.04) had protective effects approaching significance (table 4). Again, the nested multivariable models using the complete dataset and imputed missing variables showed similar results (see supplementary tables 1 and 2 available online only). Covariates with independent adverse effects on all-cause mortality after stroke were increasing age, history of MI and DM (table 3); covariates with independent adverse effects on cardiovascular mortality after stroke were increasing age and hypercholesterolaemia, while female sex had a protective effect (table 4).

The nested models revealed that the effect of healthy lifestyle practices/factors was similar regardless of whether BMI and/or the six clinical factors were included (see supplementary tables 1 and 2 available online only). In addition, BMI as an individual factor was not important regardless of the inclusion or exclusion of other variables. In general, results from the imputed and complete case analyses were qualitatively similar.

Analysis of the relationship between number of healthy lifestyle factors and all-cause mortality revealed a cumulative effect (figure 1). The rate of all-cause mortality was reduced by $96 \%$ in those who followed at least four factors versus none (HR 0.04; CI 0.01 to 0.20 ) and by $88 \%$ in those who followed $1-3$ factors versus none (HR 0.12; CI 0.03 to 0.47 ), after controlling for the other factors. Consistent with a cumulative effect, adherence to 4-5 factors was associated with significantly better mortality outcomes than adherence to only $1-3$ factors, after controlling for the other factors (HR 0.38; CI 0.22 to 0.66 ). The results were similar even after controlling for the individual health factors, including exercise and smoking (HR 0.33; CI 0.15 to 0.71). Moreover, once the number of health factors was known, smoking and exercise were no longer significant.

For cardiovascular mortality, results were similar, although slightly less robust (figure 2). The rate of cardiovascular mortality was reduced by $85 \%$ for those who followed $1-3$ 
Table 2 Bivariate analyses of predictors of all-cause and cardiovascular mortality after stroke among individuals with a self-reported history of stroke

\begin{tabular}{|c|c|c|c|c|}
\hline \multirow[b]{2}{*}{ Predictor } & \multicolumn{2}{|l|}{ All-cause mortality } & \multicolumn{2}{|c|}{ Cardiovascular mortality } \\
\hline & HR (95\% CI) & p Value & HR (95\% CI) & p Value \\
\hline Body mass index $\geq 30 \mathrm{~kg} / \mathrm{m}^{2}$ vs normal & $0.76(0.50$ to 1.16$)$ & 0.20 & $0.78(0.44$ to 1.42$)$ & 0.42 \\
\hline Body mass index $<18.5 \mathrm{~kg} / \mathrm{m}^{2}$ vs normal & $0.78(0.35$ to 1.75$)$ & 0.54 & $1.03(0.46$ to 2.28$)$ & 0.95 \\
\hline Abstinence from smoking & $1.57(0.98$ to 2.52$)$ & 0.06 & $1.70(0.89$ to 3.27$)$ & 0.11 \\
\hline$\geq 5$ servings fruits/vegetables vs none & $0.73(0.32$ to 1.68$)$ & 0.46 & $0.44(0.19$ to 1.02$)$ & 0.06 \\
\hline $1-4$ servings fruits/vegetables vs none & $0.56(0.24$ to 1.33$)$ & 0.19 & $0.30(0.12$ to 0.74$)$ & 0.009 \\
\hline Moderate vs heavy alcohol intake & $0.53(0.14$ to 2.08$)$ & 0.36 & 5.10 (0.56 to 47.62$)$ & 0.15 \\
\hline Moderate vs no alcohol intake & $0.41(0.22$ to 0.76$)$ & 0.005 & $0.51(0.25$ to 1.05$)$ & 0.07 \\
\hline Regular exercise & $0.59(0.40$ to 0.86$)$ & 0.007 & $0.69(0.41$ to 1.17$)$ & 0.17 \\
\hline Age (per year) & 1.07 (1.04 to 1.09$)$ & $<0.001$ & 1.05 (1.02 to 1.08$)$ & $<0.001$ \\
\hline Female vs male & $1.05(0.72$ to 1.52$)$ & 0.79 & $0.80(0.50$ to 1.29$)$ & 0.36 \\
\hline Hispanic vs white non-Hispanic & $0.52(0.24$ to 1.15$)$ & 0.10 & $0.49(0.16$ to 1.48$)$ & 0.20 \\
\hline Black vs white non-Hispanic & $1.14(0.79$ to 1.64$)$ & 0.47 & $0.91(0.60$ to 1.39$)$ & 0.67 \\
\hline Hypertension & 1.61 (1.05 to 2.47$)$ & 0.03 & 2.07 (1.10 to 3.87$)$ & 0.02 \\
\hline Diabetes mellitus & 1.91 (1.28 to 2.83 ) & 0.002 & 1.69 (1.08 to 2.67$)$ & 0.02 \\
\hline History of myocardial infarction & 1.59 (1.13 to 2.22$)$ & 0.008 & $1.64(1.12$ to 2.40$)$ & 0.01 \\
\hline Hypercholesterolaemia & $0.91(0.66$ to 1.25$)$ & 0.56 & $1.44(0.89$ to 2.31$)$ & 0.13 \\
\hline Hypertriglyceridaemia & $0.99(0.69$ to 1.41$)$ & 0.95 & $0.98(0.64$ to 1.51$)$ & 0.92 \\
\hline Low level of high density lipoprotein & $1.38(0.92$ to 2.06$)$ & 0.12 & $1.23(0.79$ to 1.92$)$ & 0.36 \\
\hline
\end{tabular}

health factors versus none (HR 0.15; CI 0.02 to 1.15 ) and by $92 \%$ for those who followed $4-5$ health factors versus none (HR 0.08; CI 0.01 to 0.66 ). Moreover, those who followed at least four health factors had a $47 \%$ reduction in the rate of cardiovascular mortality compared with those who followed only $1-3$ health factors (HR 0.53; CI 0.28 to 0.98). Results were similar even after controlling for the individual health factors (HR 0.42; CI 0.18 to 0.98 ).

\section{DISCUSSION}

We found that a combination of healthy lifestyle factors is associated with lower all-cause and cardiovascular mortality after stroke. Among the individual healthy lifestyle factors, only regular exercise and not smoking were independently associated with lower all-cause mortality, while eating $\geq 1$ serving of fruits/ vegetables/day was associated with a trend towards lower cardiovascular mortality after stroke, after controlling for covariates. Higher numbers of healthy lifestyle factors amplified reductions in all-cause and cardiovascular mortality. The

Table 3 Multivariable Cox hazard model* ${ }^{*}$ for predictors of all-cause mortality after stroke $(n=428)$

\begin{tabular}{llc}
\hline Predictor & HR (95\% CI) & p Value \\
\hline Normal BMI vs BMI $\geq 30 \mathrm{~kg} / \mathrm{m}^{2}$ & $0.96(0.63$ to 1.47$)$ & 0.86 \\
Normal BMI vs BMI $<18.5 \mathrm{~kg} / \mathrm{m}^{2}$ & $1.67(0.30$ to 9.09$)$ & 0.56 \\
Abstinence from smoking & $0.57(0.34$ to 0.98$)$ & 0.04 \\
$\geq 5$ servings fruits/vegetables vs none & $0.46(0.14$ to 1.47$)$ & 0.19 \\
$1-4$ servings fruits/vegetables vs none & $0.41(0.13$ to 1.35$)$ & 0.14 \\
Moderate vs heavy alcohol intake & $0.61(0.22$ to 1.69$)$ & 0.34 \\
Moderate vs no alcohol intake & $0.65(0.36$ to 1.19$)$ & 0.16 \\
Regular exercise & $0.66(0.44$ to 0.99$)$ & 0.04 \\
Age (per year) & $1.09(1.05$ to 1.12$)$ & $<0.001$ \\
Female vs male & $0.74(0.52$ to 1.07$)$ & 0.10 \\
Diabetes mellitus & $2.09(1.44$ to 3.04$)$ & $<0.001$ \\
History of myocardial infarction & $1.54(1.08$ to 2.20$)$ & 0.02 \\
\hline
\end{tabular}

*Model adjusted for: BMI, smoking, servings of fruits/vegetables, alcohol intake, exercise, age, sex, history of myocardial infarction and diabetes mellitus. BMI, body mass index. dose-response association is in accord with other studies showing a graded cardiovascular benefit of healthy lifestyle practices. 472627

This is the first study to our knowledge to assess the effect of a healthy lifestyle on mortality after stroke. Prior studies in the general population and in those with established coronary artery disease revealed that adopting a healthy lifestyle led to lower cardiovascular events, including stroke, and reduced cardiovascular and all-cause mortality. ${ }^{4-7}$ 26-30 While previous studies revealed that each healthy behaviour independently lowered the risk for cardiovascular events, ${ }^{5} 26 \quad 27$ all-cause mortality ${ }^{7}$ and cardiovascular mortality, ${ }^{7}$ our study only showed an independent effect of regular exercise and not smoking on all-cause mortality after stroke. Most studies explored the influence of healthy factors in the general population; perhaps the role of these factors in persons with established symptomatic cerebrovascular disease is different. In addition, all except one prior study ${ }^{6}$ used different definitions of healthy lifestyle practices.

Table 4 Multivariable competing risks regression model ${ }^{*}$ for predictors of cardiovascular mortality after stroke $(n=419)$

\begin{tabular}{llc}
\hline Predictor & HR (95\% CI) & p Value \\
\hline Normal BMI vs BMI $\geq 30 \mathrm{~kg} / \mathrm{m}^{2}$ & $0.85(0.46$ to 1.56$)$ & 0.61 \\
Normal BMI vs $<18.5 \mathrm{~kg} / \mathrm{m}^{2}$ & $0.77(0.14$ to 4.35$)$ & 0.76 \\
Abstinence from smoking & $0.85(0.41$ to 1.75$)$ & 0.66 \\
$\geq 5$ servings fruits/vegetables vs none & $0.30(0.09$ to 1.04$)$ & 0.06 \\
$1-4$ servings fruits/vegetables vs none & $0.30(0.08$ to 1.08$)$ & 0.07 \\
Moderate vs heavy alcohol intake & $7.14(0.68$ to 100.00$)$ & 0.10 \\
Moderate vs no alcohol intake & $0.62(0.31$ to 1.25$)$ & 0.19 \\
Regular exercise & $0.76(0.47$ to 1.23$)$ & 0.27 \\
Age (per year) & $1.07(1.03$ to 1.11$)$ & $<0.005$ \\
Female vs male & $0.48(0.29$ to 0.80$)$ & 0.004 \\
History of myocardial infarction & $1.45(0.93$ to 2.26$)$ & 0.10 \\
Hypercholesterolaemia & $1.79(1.12$ to 2.89$)$ & 0.02
\end{tabular}

*Model adjusted for: BMI, smoking, servings of fruits/vegetables, alcohol intake, exercise, age, sex, history of myocardial infarction and hypercholesterolaemia. $\mathrm{BMI}$, body mass index. 


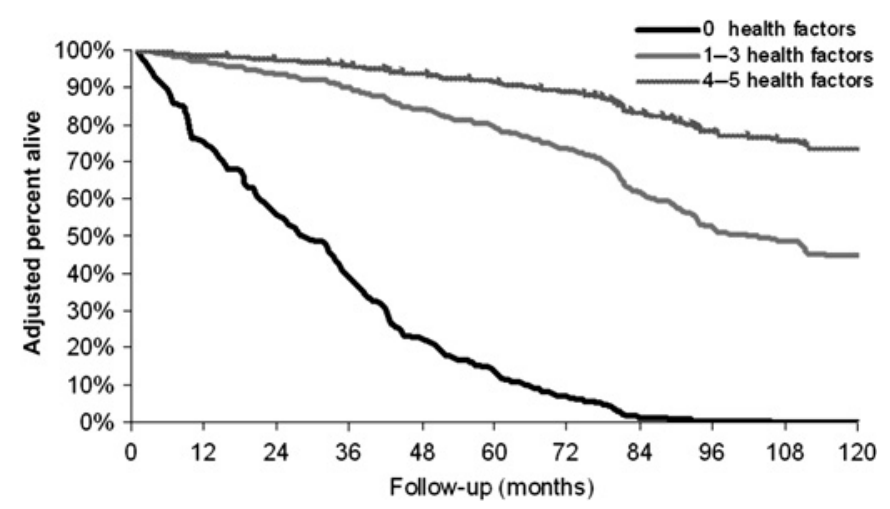

Figure 1 Protective effects of number of healthy lifestyle practices on all-cause mortality after adjustment for covariates.

Our study revealed an overall greater benefit of healthy behaviours compared with studies of primary stroke prevention ${ }^{4}$ and mortality reduction, ${ }^{6} 7$ but a similar effect size compared with studies of coronary heart disease prevention. ${ }^{26} 27$ However, different definitions of healthy lifestyle practices limit the extent to which comparisons can be made.

This study has several limitations. Firstly, since NHANES is cross sectional, participants' medical history, medication use and lifestyle practices prior to the stroke were unknown. In addition, the survey did not assess either stroke severity or post-stroke disability. These factors, which can potentially play a role in stroke mortality, were not controlled for. For example, healthy lifestyle factors may affect stroke severity which in turn affects stroke mortality. In addition, stroke severity affects the ability to adhere to lifestyle practices. Secondly, due to the cross sectional nature of the NHANES evaluation, we were only able to determine the presence or absence of healthy lifestyle factors at a time point after the stroke, without controlling for time since stroke or duration of adherence to healthy lifestyle practices. In addition, individuals' adherence to healthy lifestyle practices may have changed from the initial NHANES assessment (1988-1994) to the time of the outcomes assessment in 2000. Thirdly, NHANES relies on self-reported history of stroke, exercise frequency, alcohol use, smoking and fruit/vegetable intake. Although NHANES has not validated self-reporting of stroke, other studies found this method to have a sensitivity of $80-95 \%$ and a specificity of $96-99 \% .{ }^{31} 32$ Fourthly, the effect of healthy lifestyle practices on mortality may differ in individuals

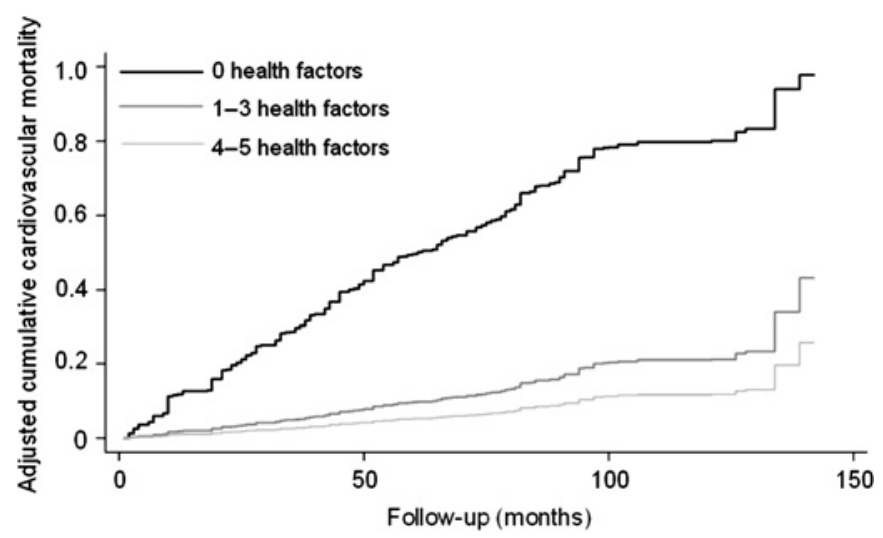

Figure 2 Protective effects of number of healthy lifestyle practices on cardiovascular mortality after adjustment for covariates. with ischaemic versus haemorrhagic strokes, and the NHANES questionnaire does not differentiate between ischaemic and haemorrhagic stroke. Finally, only $\sim 60 \%$ of stroke survivors who participated in NHANES were included in the final analysis; this relatively small number limited the power to detect effects from individual behaviours.

Nevertheless, this study implies that individuals with previous stroke have a lower risk of death from all-causes if they exhibit a higher number of healthy lifestyle factors, suggesting that interventions for improving adherence to healthy lifestyle behaviours among stroke patients may be warranted. Given the difficulties in accomplishing lifestyle change, interventions will likely require a multifaceted approach, incorporating education, social support and community involvement.

Competing interests None.

Ethics approval This was a cross sectional study performed by the Centers for Disease Control and all approvals were obtained.

Contributors AT: conception and design, analysis and interpretation of the data, drafting the article and final approval of the version to be published. DM: acquisition of the data, statistical analysis, analysis and interpretation of the data, critical revision of the manuscript for important intellectual content and final approval of the version to be published. BO: conception and design, analysis and interpretation of the data, critical revision of the manuscript for important intellectual content and final approval of the version to be published.

Provenance and peer review Not commissioned; externally peer reviewed.

\section{REFERENCES}

1. Hardie K, Hankey GJ, Jamrozik K, et al. Ten-year survival after first-ever stroke in the Perth community stroke study. Stroke 2003;34:1842-6.

2. Hartmann A, Rundek $\mathrm{T}$, Mast $\mathrm{H}$, et al. Mortality and causes of death after first ischemic stroke: the Northern Manhattan Stroke Study. Neurology 2001;57:2000-5.

3. Dennis MS, Burn JP, Sandercock PA, et al. Long-term survival after first-ever stroke: the Oxfordshire Community Stroke Project. Stroke 1993;24:796-800.

4. Kurth T, Moore SC, Gaziano JM, et al. Healthy lifestyle and the risk of stroke in women. Arch Intern Med 2006;166:1403-9.

5. Myint PK, Luben RN, Wareham NJ, et al. Combined effect of health behaviours and risk of first ever stroke in 20,040 men and women over 11 years' follow-up in Norfolk cohort of European Prospective Investigation of Cancer (EPIC Norfolk): prospective population study. BMJ 2009;338:b349.

6. King DE, Mainous AG 3rd, Geesey ME. Turning back the clock: adopting a healthy lifestyle in middle age. Am J Med 2007:120:598-603.

7. Knoops KT, de Groot LC, Kromhout D, et al. Mediterranean diet, lifestyle factors, and 10 -year mortality in elderly European men and women: the HALE project. JAMA 2004;292:1433-9.

8. Haveman-Nies A, de Groot LP, Burema J, et al. Dietary quality and lifestyle factors in relation to 10-year mortality in older Europeans: the SENECA study. Am J Epidemiol 2002;156:962-8.

9. Carlsson AC, Theobald H, Wandell PE. Health factors and longevity in men and women: a 26-year follow-up study. Eur J Epidemiol 2010;25:547-51.

10. Nechuta SJ, Shu XO, Li HL, et al. Combined impact of lifestyle-related factors on total and cause-specific mortality among Chinese women: prospective cohort study. PLoS Med 2010;7: pii:e1000339.

11. Tamakoshi A, Kawado M, Ozasa K, et al. Impact of smoking and other lifestyle factors on life expectancy among Japanese: findings from the Japan Collaborative Cohort (JACC) Study. J Epidemiol 2010;20:370-6.

12. Anon. Plan and operation of the Third National Health and Nutrition Examination Survey, 1988-94. Series 1: programs and collection procedures. Vital Health Stat 1994;32:1-407.

13. Anderson RN, Miniño AM, Hoyert DL, et al. Comparability of cause of death between ICD-9 and ICD-10: preliminary estimates. Nat/ Vital Stat Rep 2001:49:1-32.

14. King DE, Mainous AG 3rd, Carnemolla M, et al. Adherence to healthy lifestyle habits in US adults, 1988-2006. Am J Med 2009:122:528-34.

15. Sacco RL, Adams R, Albers G, et al. Guidelines for prevention of stroke in patients with ischemic stroke or transient ischemic attack: a statement for healthcare professionals from the American Heart Association/American Stroke Association Council on Stroke: co-sponsored by the Council on Cardiovascular Radiology and Intervention: the American Academy of Neurology affirms the value of this guideline. Stroke 2006;37:577-617.

16. Goldstein LB, Adams R, Alberts MJ, et al. Primary prevention of ischemic stroke: a guideline from the American Heart Association/American Stroke Association Stroke Council: cosponsored by the Atherosclerotic Peripheral Vascular Disease Interdisciplinary Working Group; Cardiovascular Nursing Council; Clinical Cardiology Council; Nutrition, Physical Activity, and Metabolism Council; and the Quality of Care and Outcomes Research Interdisciplinary Working Group: the American Academy of Neurology affirms the value of this guideline. Stroke 2006;37:1583-633. 
17. Djuric Z, Ren J, Mekhovich 0, et al. Effects of high fruit-vegetable and/or low-fat intervention on plasma micronutrient levels. J Am Coll Nutr 2006;25:178-87.

18. Magarey A, McKean S, Daniels L. Evaluation of fruit and vegetable intakes of Australian adults: the National Nutrition Survey 1995. Aust N Z J Public Health 2006;30:32-7.

19. Forman D, Bulwer BE. Cardiovascular disease: optimal approaches to risk factor modification of diet and lifestyle. Curr Treat Options Cardiovasc Med 2006;8:47-57.

20. Dietary Guidelines for Americans 2005, Executive Summary. http://www.health.gov/ dietaryguidelines (accessed 10 Jan 2010).

21. Haskell WL, Lee IM, Pate RR, et al. Physical activity and public health: updated recommendation for adults from the American College of Sports Medicine and the American Heart Association. Circulation 2007;116:1081-93.

22. Tanasescu M, Leitzmann MF, Rimm EB, et al. Exercise type and intensity in relation to coronary heart disease in men. JAMA 2002;288:1994-2000.

23. Anon. American College of Sports Medicine Position Stand. The recommended quantity and quality of exercise for developing and maintaining cardiorespiratory and muscular fitness in healthy adults. Med Sci Sports Exerc 1990;22:265-74.

24. Flegal KM, Graubard BI, Williamson DF, et al. Excess deaths associated with underweight, overweight, and obesity. JAMA 2005;293:1861-7.

25. US Department of Health and Human Services and US Department of Agriculture. Alcoholic beverages. In: Dietary Guidelines for Americans. 2005.
http://www.health.gov/DIETARYGUIDELINES/dga2005/document/html/chapter9.htm (accessed 10 Jan 2010)

26. Stampfer MJ, Hu FB, Manson JE, et al. Primary prevention of coronary heart disease in women through diet and lifestyle. N Engl J Med 2000;343:16-22

27. Chiuve SE, McCullough ML, Sacks FM, et al. Healthy lifestyle factors in the primary prevention of coronary heart disease among men: benefits among users and nonusers of lipid-lowering and antihypertensive medications. Circulation 2006; 114:160-7

28. Appel LJ, Champagne CM, Harsha DW, et al. Effects of comprehensive lifestyle modification on blood pressure control: main results of the PREMIER clinical trial. JAMA 2003;289:2083-93.

29. Iestra J, Knoops K, Kromhout D, et al. Lifestyle, Mediterranean diet and survival in European post-myocardial infarction patients. Eur J Cardiovasc Prev Rehabil 2006; 13:894-900.

30. O'Donnell MJ, Xavier D, Liu L, et al. Risk factors for ischaemic and intracerebral haemorrhagic stroke in 22 countries (the INTERSTROKE study): a case-control study. Lancet 2010;376:112-23.

31. Engstad T, Bonaa KH, Viitanen M. Validity of self-reported stroke: The Tromso Study. Stroke 2000;31:1602-7.

32. O'Mahony PG, Dobson R, Rodgers $\mathrm{H}$, et al. Validation of a population screening questionnaire to assess prevalence of stroke. Stroke 1995;26:1334-7. 